\title{
Computer Modeling of Aerosol Emissions Spread in the Atmosphere
}

\author{
Daler Sharipov ${ }^{1, *}$, Sharofiddin Aynakulov ${ }^{2}$ and Otabek Khafizov ${ }^{2}$ \\ ${ }^{1}$ Department of Multimedia Technologies, university of information technologies named after \\ Muhammad al-Khwarizmi, Tashkent, 100000, Uzbekistan \\ ${ }^{2}$ Department of Information Technologies, Tashkent institute of irrigation and agricultural \\ mechanization engineers, Tashkent, 100000, Uzbekistan
}

\begin{abstract}
The paper deals with the development of mathematical model and numerical algorithms for solving the problem of transfer and diffusion of aerosol emissions in the atmospheric boundary layer. The model takes into account several significant parameters such as terrain relief, characteristics of underlying surface and weather-climatic factors. A series of numerical experiments were conducted based on the given model. The obtained results presented here show how these factors affect aerosol emissions spread in the atmosphere.
\end{abstract}

\section{Introduction}

An intensive growth of production and the expansion of transport systems in the world have considerable influence on the ecological condition of the environment. This is particularly evident for developing countries which are distinctive by a high rate of establishing new production facilities. The increase of harmful substances emission into the atmosphere inevitably involves an imbalance in the ecological condition of industrial regions with possible climatic changes.

In this respect, such issues as the assessment of air pollution and contamination of underlying surface by passive and active aerosol emissions, the deployment of new industrial facilities in compliance with sanitary norms, the determination of intensity and level of air and water pollution and the influence of emissions on flora and fauna are relevant in environment protection problem. And not only for individual regions, but also worldwide.

However, the restrictive conservation measures should not adversely affect the economic and industrial growth that makes the environment protection problem very actual and requiring accurately weighed decisions.

Computer modeling is one of effective means of decision-making support. Plenty applied and fundamental researches are conducted worldwide by the use of computer modeling of transfer and diffusion harmful substances in the atmosphere.

Though, the analysis of publications over recent years showed that most authors did not consider the process of transport and diffusion of aerosol emissions in the atmosphere as

\footnotetext{
* Corresponding author: qushkor@ rambler.ru
} 
three-dimensional problem and did not take into account such important parameter as the coefficient of absorption of harmful substances in the atmosphere that varies in days and seasons.

Also, it should be noted that when modeling the considered process many authors assumed that the spread of harmful substances did not reach defined boundaries of solution region and there was no influx of harmful substances through these boundaries. But conducted researches of this process showed that with big values of wind speed there occurs the transport of aerosols over the boundaries of problem solution region.

Within this paper, certain efforts have been made to fill mentioned gap. Pursuant to foregoing, the purpose of this work is to develop mathematical model and numerical algorithm for solving the problem of transfer and diffusion of aerosol emissions in the atmospheric boundary layer.

\section{Problem statement}

The mathematical model of the spread of harmful substances in the atmospheric boundary layer taking into account terrain orography has following form:

$$
\begin{aligned}
& \frac{\partial \theta(x, y, z, t)}{\partial t}+\frac{\partial}{\partial x}(u H(x, y, z) \theta(x, y, z, t))+\frac{\partial}{\partial y}(v H(x, y, z) \theta(x, y, z, t))+ \\
& +\frac{\partial}{\partial z}\left(\left(w-w_{g}\right) H(x, y, z) \theta(x, y, z, t)\right)+\sigma \theta(x, y, z, t) H(x, y, z)= \\
& =\mu \frac{\partial}{\partial x}\left(H(x, y, z) \frac{\partial \theta(x, y, z, t)}{\partial x}\right)+\mu \frac{\partial}{\partial y}\left(H(x, y, z) \frac{\partial \theta(x, y, z, t)}{\partial y}\right) \\
& +\frac{\partial}{\partial z}\left(\lambda H(x, y, z) \frac{\partial \theta(x, y, z, t)}{\partial z}\right)+\delta_{i, j, k} f(x, y, z, t), \\
& \left.\theta(x, y, z, t)\right|_{t=0}=\theta_{0}(x, y, z), \\
& \left.\alpha_{1}\left(\theta(x, y, z, t)-\theta_{0}\right)\right|_{x=0}+\left.\beta_{1} \frac{\partial \theta}{\partial x}\right|_{x=0}=0 \\
& \left.\alpha_{2}\left(\theta(x, y, z, t)-\theta_{H}\right)\right|_{x=L}+\left.\beta_{2} \frac{\partial \theta}{\partial x}\right|_{x=L_{x}}=0 \\
& \left.\alpha_{3}\left(\theta(x, y, z, t)-\theta_{H}\right)\right|_{y=0}+\left.\beta_{3} \frac{\partial \theta}{\partial y}\right|_{y=0}=0 \\
& \left.\alpha_{4}\left(\theta(x, y, z, t)-\theta_{H}\right)\right|_{y=L_{y}}+\left.\beta_{4} \frac{\partial \theta}{\partial y}\right|_{y=L_{y}}=0 \\
& \lambda \frac{\partial \theta}{\partial z}-H \beta \theta=-H f_{0} \text { when } z=0, \\
& \lambda \frac{\partial \theta}{\partial z}=0 \text { when } z=H_{z} .
\end{aligned}
$$


Here $\theta$ - concentration of harmful substances in the atmosphere; $\theta_{0}$ - initial concentration of harmful substances in the atmosphere; $x, y, z$ - coordinate system; $u, v, w$ - wind speed in three directions; $w_{g}$ - rate of particles settling; $\sigma$ - coefficient of harmful substances absorption in the atmosphere; $\mu, \lambda$-coefficients of diffusion and turbulence; $\delta_{i, j, k}$ - Dirac function; $f$ - harmful substances emitter situated on the underlying surface. The parameters $\alpha_{1}, \alpha_{2}, \alpha_{3}, \alpha_{4}, \beta_{1}, \beta_{2}, \beta_{3}, \beta_{4}$ in Eq. 1 - Eq. 6 are defined depending on problem statement and can be equal to 0 or 1 .

In this statement the terrain orography and physical and mechanical characteristics of underlying earth surface are accounted by parameters $H(x, y, z)$ and $\beta(x, y, z)$. Here $H$ can be equal to 0 - if the layer is under the ground, to 1 - if the layer is in the atmosphere, and to $\left(\eta-z_{K-0,5}\right) / \Delta z$ - if the layer is under orographic surface. $\eta-$ is the height of a hill on the plane which is parallel to the sea level and $\Delta z=z_{k+0,5}-z_{k-0,5}$. For each layer of the model we introduced a multiplier $H(0 \leq H \leq 1)$ determining the degree of air flow blockage Fig. 1.

The main parameters of the model are to determine as power functions

$$
\begin{aligned}
& u(x, t)=\left|v_{1}\right|\left(\frac{z}{z_{1}}\right)^{n} \cos \beta, v(z, t)=\left|v_{1}\right|\left(\frac{z}{z_{1}}\right)^{n}, w(z, t)=\left|v_{1}\right|\left(\frac{z}{z_{1}}\right)^{n} \sin \beta, \\
& \lambda(z, t)=\lambda_{0}(z, t)+\bar{\lambda}(z, t), \mu(z, t)=\bar{\mu}|v(z, t)|,
\end{aligned}
$$

where $\left|v_{1}\right|$ is a modulus of wind speed when $z=1 \mathrm{~m}$.

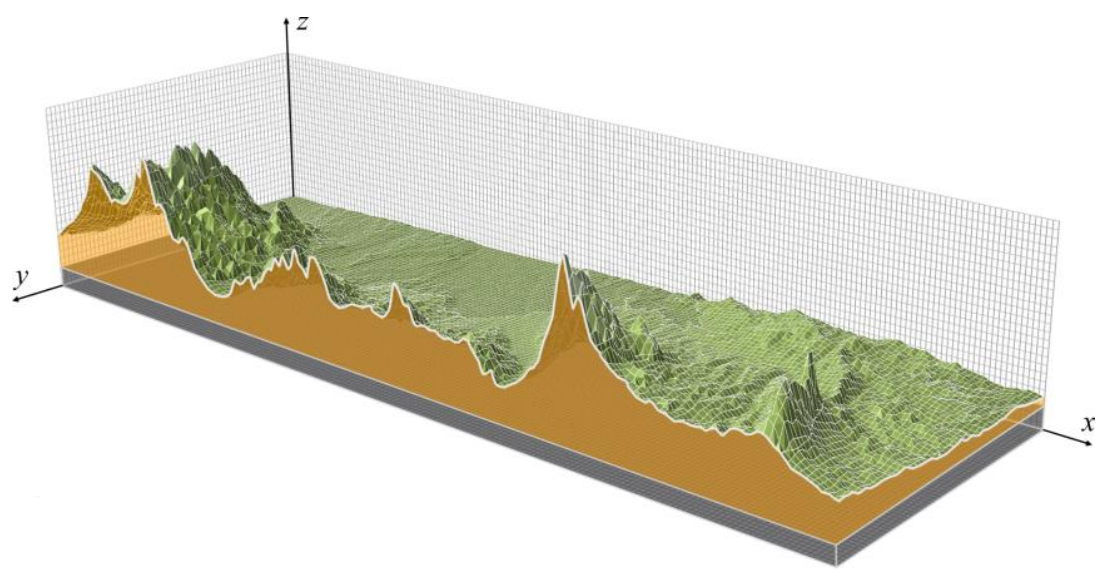

Fig. 1. The relief of terrain along aerosol emissions transport area.

- $H=\left(\eta-z_{K-0,5}\right) / \Delta z, \square-H=0, \square-H=1$, $\square$ - orographic surface.

\section{Methods}

As we can see from the problem statement Eq. 1 - -q. 8, it is too difficult to obtain an analytical solution. Therefore, for the numerical integration of the problem there was 
developed a numerical algorithm based on the substitution of differential operators on finite-difference [1-4].

Also we can see that the main factors and parameters which significantly affect the progress of the spread of harmful substances into the environment by temporary and spatial variables are the coefficients of diffusion, absorption and interaction with the earth's'surface, as well as emitters' 'ntensity and their location. It should be noted that the above factors are dependent on climatic factors, the time of day and seasons of year.

In this regards, on the basis of statistical processing of long-term data, there was obtained a sinusoidal dependence for determining the coefficient of absorption of harmful substances in the atmosphere which has following form:

$$
\sigma(t)=\sigma_{0}+\Delta \sigma \operatorname{Sin} \omega t
$$

Here $\sigma_{0}$ - daily average change of the coefficient of aerosol emissions absorption in the atmosphere; $\Delta \sigma$ - amplitude of the absorption coefficient change for a day; $\omega-$ cyclic frequency of daily change.

Dunes, hills and ridges that extend above the surrounding terrain play an important role in the changing of wind direction and speed. If the air mass meets a steep hill with a rough surface, the wind speed increases dramatically, which leads to an increase in the coefficient of turbulence Fig. 2b. Thereby, the natural barriers significantly affect the process of transfer and diffusion of pollutants into the atmosphere[5].

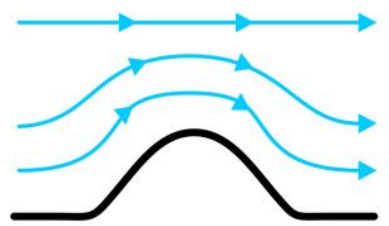

a)

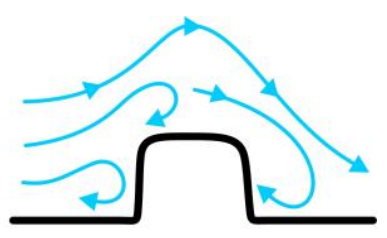

b)

Fig. 2. Air mass flow.

As the high pressure area actually extends some distance to the hill, the wind changes its direction before reaching the hill. Wind speed increases with the difference of atmospheric pressure, but the air flow rate decreases due to friction on underlying surface roughness. Therefore, the wind speed is higher over the hills than over surrounding plain area.

There were numerous attempts to identify the relationship between the content of pollutants in urban air and wind speed. Many authors pointed out that most of the air pollution thrown out by low emitters was observed at slow winds ( 0 to $1 \mathrm{~m} / \mathrm{s}$ ). When emissions were occured by high emitters, the maximal concentrations of pollution were fixed at dangerous wind speeds ( 3 to $6 \mathrm{~m} / \mathrm{s}$ depending on the emission flow rate from exhaust pipes) [6-7].

Dangerous winds coupled with unstable stratification and intense transfer of impurities lead to the maximum increase in the concentrations of harmful substances in the atmospheric boundary layer. It was brought out that in such cases the horizontal air streams play a principal role in the dissipation of pollutants.

In the course of numerical experiments with real data, the source of values for model parameters is needed. These values should be relevant for considered territory and current time. For this purpose, we developed program interfaces to communicate with geoinformation and weather web-services. For example, $H$ values for considered area are loaded online via Google Elevation API. The weather data such as wind direction and speed 
$(u, v, w)$ is loaded from several web-services «Weather Underground», «World Weather Online», «WebserviceX.NET» etc.

Also there were created auxiliary databases containing the SRTM topographic data and average annual weather characteristics for several industrial regions of Uzbekistan.

\section{Results}

In order to accomplish a comprehensive study of the process using this mathematical provision we developed a software tool, which consists of several modules for: data prepairing and entry; calculating main variables of the model; storing results of numerical calculations; interpretation and analysis of results in the form of graphics, animation; generating relief maps using available SRTM data.

Together with the real data from Weather web-services the software provides opportunity to carry out real time monitoring and forecasting the process of aerosol emissions spread in the atmosphere.

Using developed mathematical and software tools, we conducted a series of numerical experiments. The experiments were conducted for weather and climate conditions of «Akhangaran-Almalyk-Angren» region, taking into account the terrain, the values of the air mass velocity horizontally and vertically, the coefficients of absorption of harmful substances and their interaction with the underlying surface. Colors palette in the figures corresponds to the levels of carbon monoxide concentration in the atmosphere. At each time step the calculation accuracy is controlled by the material balance equation Fig. 3 Fig. 4.

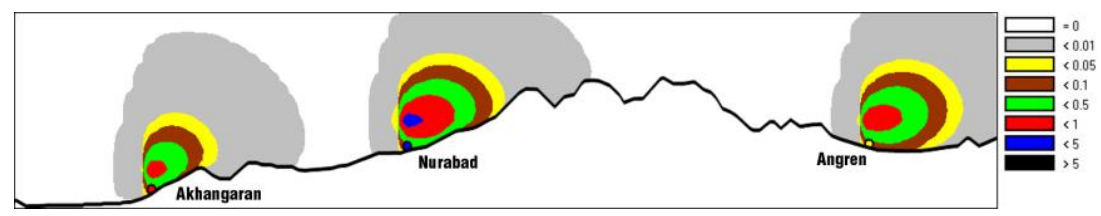

Fig. 3. Distribution of carbon monoxide concentration in the atmosphere after 1 hour.

Horizontal wind speed $-V=1 \mathrm{~m} / \mathrm{s}$. Particles settling velocity $-\mu=0,0064 \mathrm{~m} / \mathrm{s}$.

Coefficient of diffusion $-\mu=0,01$. Coefficient of turbulence $-\lambda=0,02$.

Coefficients of absorption $-\sigma=0,00062$. Time $-t=1 \mathrm{~h} . \Delta t=0,01$.

Solution region length $-L x=50 \mathrm{~km} . \Delta x=0,5$ or $500 \mathrm{~m}$.

Solution region height $-L z=2 \mathrm{~km} . \Delta z=0,1$ or $100 \mathrm{~m}$.

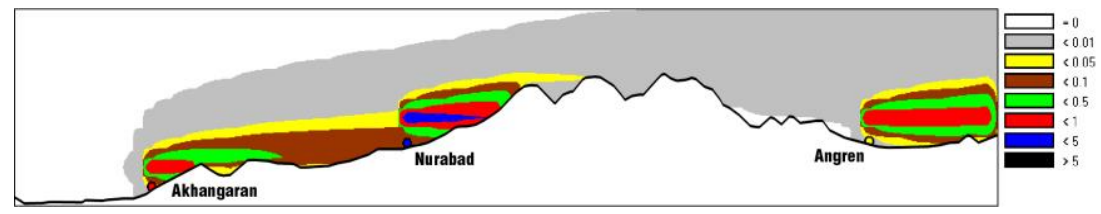

Fig. 4. Distribution of carbon monoxide concentration in the atmosphere after 1 hour.

Horizontal wind speed $-V=10 \mathrm{~m} / \mathrm{s}$.

Other parameters as in Fig. 3.

As we can see from Fig. 3 - Fig. 4. the distribution of air pollutants significantly depends on: the terrain where there are emissions from industrial facilities; vertical and horizontal velocity of the air mass; the coefficient of absorption of harmful substances in 
the atmosphere depending on the humidity, which in turn depends on the time of day and season.

The obtained results showed that with the increase of horizontal wind speed the pollutants dispersion area in the atmospheric boundary layer increases too (Fig. 4). The rate of deposition of particles on the underlying surface mainly depends on the size of aerosol particles and the vertical velocity of the ascending air. The quantity of settled particles depends on the difference in the particles settling velocity and vertical wind speed $\left(w-w_{g}\right)$.

As it was shown by numerical calculations, the maximum absorption of aerosol particles takes place in the morning and evening hours. It was determined that if there is continuous emission of harmful substances from industrial facilities into the atmosphere, a significant change in their concentration will take place precisely due to the absorption coefficient. If there are moderate winds in the «Akhangaran-Almalyk-Angren» industrial region then there will be the accumulation of harmful substances which draws the exceeding of pollution standard.

It was determined that dispersing of harmful substances in the atmosphere was significantly influenced by both horizontal and vertical velocity of air mass. Large values of the vertical velocity of the air mass cause chaotic turbulent particles dissipation. With the increase of horizontal wind speed $(V>12 \mathrm{~m} / \mathrm{s})$ particles are carried away beyond the boundaries of solution region (Fig. 5).

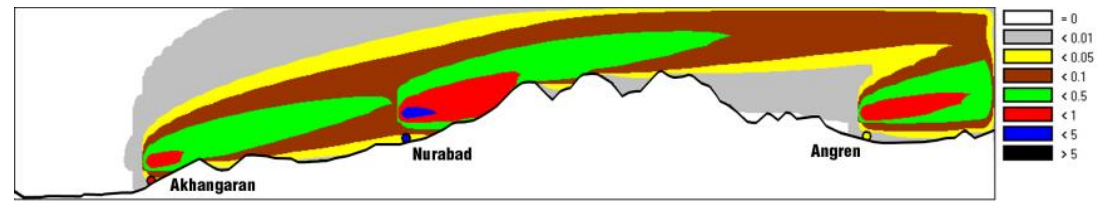

Fig. 5. Distribution of carbon monoxide concentration in the atmosphere after 1 hour.

Horizontal wind speed $-V=12 \mathrm{~m} / \mathrm{s}$. Vertical wind speed $-W=1 \mathrm{~m} / \mathrm{s}$.

Other parameters as in Fig. 3.

The obtained results favorably differ because of the fact that here the weather and climatic factors and mountain-valley air circulation features above Central Asia are taken into account.

\section{Summary}

The analysis of comparison the discussed results with experimental information confirms the adequacy of the developed model.

Developed mathematical and software tools can be used for optimal placement of newly built industrial facilities, estimating scale and geographical distribution of emissions into the environment, determining concentration of harmful substances in the atmosphere and on the underlying surface with the view of making certain decisions to minimize threats.

Ecologists can apply considered tools to monitor and predict the process of transfer and diffusion of industrial emissions, taking into account local climatic factors, orography and long-term weather data. Also they are able to determine the potential of absorption and concentration of harmful substances in certain region with regards to pollutants' impact on the flora, fauna and people. 


\section{References}

1. N. Ravshanov, M. Shertaev, N. Toshtemirova, American Journal of Modeling and Optimization. 2, 6 (2015)

2. N. Ravshanov, D.K. Sharipov, D. Ahmedov, Information technologies of modeling and management. 3, 6 (2015)

3. N. Ravshanov, D.K. Sharipov, Intellectual Archive. 6, 5 (2013)

4. D. Sharipov, ISJ Theoretical \& Applied Science, 08, 6 (2016)

5. N. Ravshanov, D. K. Sharipov, F. Muradov, International Scientific Journal "Theoretical \& Applied Science", 3, 5 (2016)

6. V.G. Gitis, E.N. Petrova, S.A.Pirogov, E.F.Yurkov, Automatics and Telemechanics, 9, (2007)

7. N. Ravshanov, D.K. Sharipov, F.A. Muradov, Uzbek journal "Problems of Informatics and Energy", 3, 8, (2016) 